\title{
Dynamic Probabilistic Atlas of Functional Brain Regions for Transcranial Magnetic Stimulation
}

\author{
Juha Koikkalainen ${ }^{1}$, Mervi Könönen ${ }^{2,4}$, Jari Karhu ${ }^{3}$, Jarmo Ruohonen ${ }^{3}$, \\ Eini Niskanen ${ }^{2,5,6}$, and Jyrki Lötjönen ${ }^{1}$ \\ ${ }^{1}$ VTT, P.O.B. 1300, FIN-33101 Tampere, Finland \\ 2 NBS-laboratory, Department of Clinical Neurophysiology, Kuopio University \\ Hospital, P.O.B. 1777, FIN-70211 Kuopio, Finland \\ ${ }^{3}$ Nexstim Ltd., Elimäenkatu 9 B, FIN-00510 Helsinki, Finland \\ ${ }^{4}$ Department of Clinical Radiology, Kuopio University Hospital, P.O.B. 1777, \\ FIN-70211 Kuopio, Finland \\ ${ }^{5}$ Department of Physics, University of Kuopio, P.O.B. 1627, FIN-70211 Kuopio, \\ Finland \\ ${ }^{6}$ Department of Neurology, Kuopio University Hospital, P.O.B. 1777, FIN-70211 \\ Kuopio, Finland
}

\begin{abstract}
Transcranial Magnetic Stimulation (TMS) is a technique to stimulate the brain non-invasively. The applications range from accurate localization of the primary motor areas to potential treatment of disorders such as tinnitus, severe depression, and pain. Stereotactic guidance requires individual MR images of the subject's head, which is in some applications typically omitted due to financial motivations. In this paper, we introduce a method that offers improved TMS pulse targeting also to those subjects who do not have MR examinations. A probabilistic brain model was constructed by spatially normalizing the locations of the functional brain areas in a study population, and modeling the distributions and estimates of the locations of the functional brain regions using probabilistic methods. The application of the probabilistic brain model to the target subject was based on a point set determined from the scalp and facial skin of the target subject. The methods were evaluated using data from four functional brain areas from 56 healthy subjects. The accuracy of the estimates of the locations of the functional brain regions was about nine millimeters.
\end{abstract}

\section{Introduction}

Transcranial Magnetic Stimulation (TMS) is a technique to stimulate the brain non-invasively [12]. The applications range from accurate localization of the primary motor areas to potential treatment of disorders such as tinnitus, severe depression, and pain 34. The clinical usefulness of TMS is greatly improved when it is combined with Magnetic Resonance (MR) imaging-guided navigation. Such combined use can tell exactly which locations of the brain are stimulated in TMS examinations [5]. Stereotactically guided TMS has been termed as Navigated Brain Stimulation (NBS).

D. Metaxas et al. (Eds.): MICCAI 2008, Part I, LNCS 5241, pp. 543 550, 2008.

(C) Springer-Verlag Berlin Heidelberg 2008 
Stereotactic guidance requires individual MR images of the target subject's (the subject for whom the NBS study is performed) head. In some of the applications of NBS, especially in psychiatric applications, the patients typically do not undergo an MR examination for diagnostic reasons. In these applications, the acquisition of the MR images is typically limited by financial motivations. In this paper, we introduce a method that offers improved TMS pulse targeting also to those groups of subjects who do not routinely have MR examinations. The objective is to provide accurate, fast, and low-cost methods to give a priori information on the locations and distributions of functional brain regions. This a priori information is useful also when MR images are available.

The main principle of the methods studied was to probabilistically model the distributions of the locations of functional brain regions by combining data from 56 subjects, for which the locations of the hand and leg motor areas were mapped with NBS. A probabilistic functional brain map was constructed by spatially normalizing the NBS localization data to the coordinate system of a mean brain template. When a target subject without MR images enters an NBS examination, the probabilistic atlas, including the mean grayscale brain template, its segmentation and functional brain map, is non-rigidly transformed to match his or her head's external shape. The non-rigid transformation is based on a set of points determined from the scalp and facial skin of the target subject. Therefore, expensive and time-consuming anatomical imaging of the target subject is not needed.

Our probabilistic atlas can be used during NBS examinations in two ways: 1) individualized head model is obtained for the target subject by deforming the head model of the mean brain template. This enables the visualization of the distributions of the locations of the functional brain regions and the current location of stimulus on a subject-specific head model, either using grayscale data of the model or surfaces made from its segmentation. 2) The probabilistic functional brain map indicates the most probable regions for stimulating desired cortical areas. When the first target location is searched, the map is based solely on the previously analyzed data from 56 subjects. However, when information about the locations of different functional brain regions becomes available during the stimulation procedure, the functional brain map is updated dynamically. In other words, the probability for a specific cortical area does not depend only on the data from the study population, but also on the already located areas of the current target subject. While this paper focuses on TMS, the methods are readily applicable also to other electromagnetic brain imaging methods such as magnetoencephalography (MEG).

\section{Materials}

The study population consisted of 56 healthy subjects (29 females, 27 males, mean age $48 \pm 16$ years, range $20-80$ years). NBS studies were performed for each subject to locate the hand and leg motor areas.

The stimulation setup consisted of the navigation system (Nexstim Ltd, Helsinki, Finland) combined with a magnetic stimulator (Nexstim Ltd, Helsinki, 
Finland) and a $50 \mathrm{~mm}$ figure-of-eight biphasic TMS coil. During stimulation, muscle activity was recorded and monitored continuously by electromyography (EMG) (ME 6000, Mega Electronics Ltd, Kuopio, Finland). EMG was measured from the thenar and hypothenar muscles (opponens pollicis) when examining hand motor area on the cortex, and from the tibialis muscle when examining leg motor area on the cortex. The NBS system delivered trigger pulses that synchronized the TMS and EMG systems.

Three anatomical landmarks and 22 marker points were defined on the scalp and facial skin. The anatomical landmarks were the nasion and the left and right peri-auricular points. The marker points were defined as follows: six points approximately equidistantly on the mid-sagittal slice starting from the tip of the nose and ending to the back of the head, six points on the horizontal slice at the level of the nasion, six points on the horizontal slice at the level of forehead, and four points on the horizontal slice at the level of the top of the brain. The difference between landmarks and marker points was that the landmarks could be defined from the same exact anatomical location from each subject, whereas the locations of the marker points could slightly vary between subjects. The points for one subject are visualized in Fig. 1] These points had two roles: 1) they allowed the reconstruction of the geometry of the head when anatomical images were not available and 2) they guided the registration processes needed to construct the probabilistic model of the locations of the functional brain regions and to match this model to the coordinate system of the target subject.
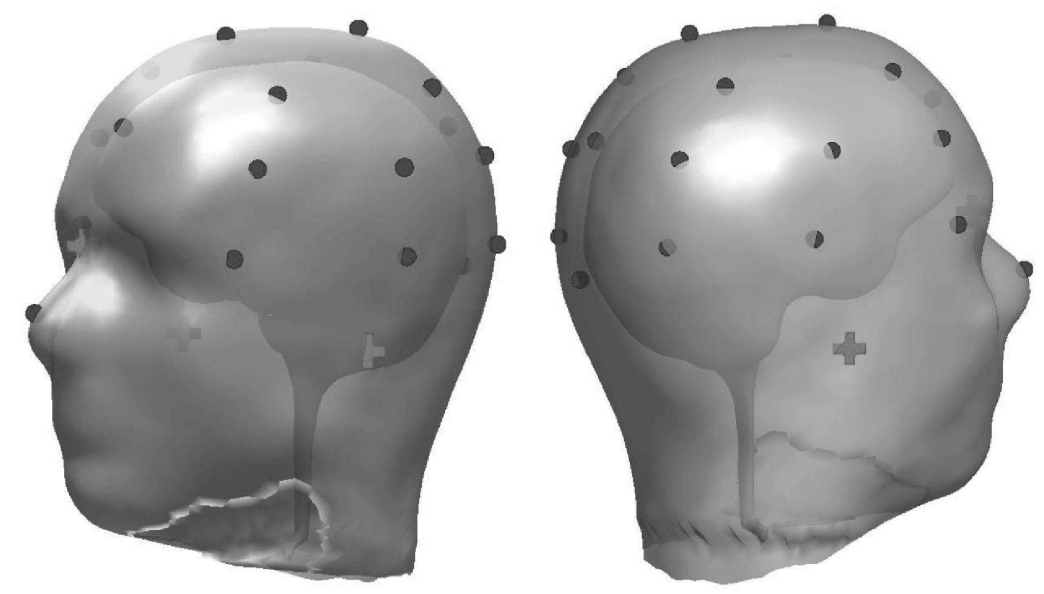

Fig. 1. The surfaces of the probabilistic atlas and the markers (spheres), and the anatomical landmarks (crosses)

\section{Methods}

The probabilistic brain model consists of a mean brain template representing the average brain anatomy and distributions and estimates of the locations of 
functional brain areas. In the NBS studies, the constructed probabilistic brain model is transformed into the target subject head providing the estimates of the locations of the functional brain areas for the target subject and enabling the visualization of the distributions of the functional brain areas on a subjectspecific head model.

\subsection{Construction of Probabilistic Brain Model}

Mean Brain Template. A mean brain template representing the average anatomy of the healthy population was used as the reference in this study. The template had been constructed in the previous studies using the MR images of 31 healthy subjects (not included in the study population of this study) using methods presented in [6]. An MR volume representing the mean brain anatomy was generated from the MR images. This volume can be used in visualizing different spatial data related to NBS, such as the locations of functional regions, in subject specific anatomy. The surfaces representing the scalp, skull, and brain envelope, and the anatomical landmarks were also determined manually.

Registration of Training Set Data to Mean Brain Template. The study population has to be spatially normalized to study the distributions of the locations of functional brain areas. The normalization was performed by registering the study population subjects to the mean brain template.

The point-based registration method used in this work was originally presented in [6]. The method was composed of three steps: rigid, affine, and nonrigid registrations. First, the anatomical landmarks of the mean brain template were rigidly registered to the anatomical landmarks of the study population subject using the method proposed in 7]. Then, the registration was continued using 9-parameter affine transformation and all the anatomical landmarks and marker points. Finally, the scalp surface of the mean brain template was registered non-rigidly to the marker points of the study population subject using the free-form deformation (FFD) grid with linear basis functions and grid size $4 \times 4 \times 4$ [ $]$. The 9 -affine and FFD transformations were optimized by minimizing a weighted sum of the mean distance from the markers of the subject, $\mathbf{p}_{i}$, to the scalp surface of the mean brain template, $\mathbf{M}$, and the distance between the corresponding anatomical landmarks of the subject, $\mathbf{l}_{i}$, and the mean brain template, $\mathbf{m}_{i}$ :

$$
E=\frac{1}{N_{s}} \sum_{i=1}^{N_{s}} d\left(\mathbf{M}, \mathbf{p}_{i}\right)+\alpha \frac{1}{N_{l}} \sum_{i=1}^{N_{l}}\left\|\mathbf{l}_{i}-\mathbf{m}_{i}\right\| .
$$

The number of the anatomical landmarks and marker points were denoted by $N_{l}$ and $N_{s}$, respectively. The distance $d()$ was defined from a distance map computed for the scalp surface. In this study, the optimal values for the weight $\alpha$ were searched by testing several values. The values used were $\alpha=1$ in affine registration and $\alpha=0.05$ in FFD registration.

Distributions and Estimates of Functional Brain Areas. After the NBS localization results of each study population subject had been transformed into 
the coordinate system of the mean brain template, the distributions of the functional brain areas were determined. These distributions give good first estimates for the locations of functional brain areas so that only small adjustments are needed to find the optimal locations during the stimulation.

The distributions of the functional brain areas were estimated using nonparametric probability density functions (Parzen windowing [9]). The probability that the $j$ th functional brain area was located at location $(x, y, z)$ was computed as

$$
\begin{gathered}
\hat{P}_{j}(x, y, z)=\sum_{i=1}^{N} \frac{1}{\sqrt{2 \pi \sigma_{1}^{2}}} e^{-\frac{\left\|(x, y, z)-X_{i, j}\right\|^{2}}{2 \sigma_{1}^{2}}}, \\
P_{j}(x, y, z)=\frac{\hat{P}_{j}(x, y, z)}{\sum_{x, y, z} \hat{P}_{j}(x, y, z)},
\end{gathered}
$$

where $N$ was the number of subjects, $X_{i, j}$ the location of the $j$ th functional brain area of the $i$ th subject, and $\sigma_{1}$ was used to control the width of the Gaussian window. In this study, we used $\sigma_{1}=5 \mathrm{~mm}$. The normalization in Eq. 3 was done so that the probabilities would sum up to one. Similarly, the mean of the locations of a functional brain area in the study population was used as the estimate of the location of the $j$ th functional brain area $\hat{Y}_{j}$ :

$$
\hat{Y}_{j}=\frac{1}{N} \sum_{i=1}^{N} X_{i, j} .
$$

In the NBS studies, the hand motor area is usually located first, and after that the motor area of leg. In this study, a technique was proposed in which the optimal location of a functional brain area determined with NBS was utilized to improve the accuracy of the distribution and estimate of another functional brain area. It was hypothesized that if, for example, the motor area of hand of a study population subject is near to the corresponding area of the target subject the same is true also for the motor area of leg.

Based on this hypothesis, a Gaussian model was assumed for the dependency between the locations of different functional brain areas. Weights were determined for each study population subject based on the distance from the location of a functional brain area of the study population subject to the optimal location of the target subject obtained with NBS. A Gaussian function was used to determine the weights:

$$
w_{i, j}=\frac{1}{\sqrt{2 \pi \sigma_{2}^{2}}} e^{-\frac{\left\|X_{i, j}-Y_{j}\right\|^{2}}{2 \sigma_{2}^{2}}},
$$

where $Y_{j}$ was the real location of the $j$ th functional brain area of the target subject obtained with NBS and $\sigma_{2}$ was used to control the width of the Gaussian function. With the small values of $\sigma_{2}$, those study population subjects for which $\left\|X_{i, j}-Y_{j}\right\|$ is small will get large weights. If $\sigma_{2}$ is large, also those subjects, for 
which $\left\|X_{i, j}-Y_{j}\right\|$ is large, will have moderate weights. The optimal value for $\sigma_{2}$ was searched and used in computing the results.

If the optimal localization results of several functional brain areas were used, the weights of all the areas were multiplied,

$$
\hat{w}_{i}=\prod_{j} w_{i, j},
$$

and, normalized so that they summed up to one:

$$
\bar{w}_{i}=\frac{\hat{w}_{i}}{\sum_{j=1}^{N} \hat{w}_{j}} .
$$

Finally, the distribution of the functional brain area was approximated using the weighted version of Eq. 2.

$$
P_{j}(x, y, z)=\sum_{i=1}^{N} \bar{w}_{i} \frac{1}{\sqrt{2 \pi \sigma_{1}^{2}}} e^{-\frac{\left\|(x, y, z)-X_{i, j}\right\|^{2}}{2 \sigma_{1}^{2}}},
$$

and the estimate of the location of the functional brain area was computed as the weighted mean of the locations of the functional brain area in the study population:

$$
\hat{Y}_{j}=\sum_{i=1}^{N} \bar{w}_{i} X_{i, j}
$$

\subsection{Evaluation}

The accuracy of the estimates of the locations of functional brain areas was evaluated by measuring the Euclidean distances from the estimates $\hat{Y}_{j}$ to the real locations of the functional brain regions $Y_{j}$ obtained using NBS. This was performed for all the subjects, and the mean of the estimation errors was computed for each functional brain region. Three techniques of determining the estimate were evaluated: Technique 1) The mean of the study population locations (Eq. (4). Technique 2) The weighted mean of the study population locations (Eq. 9) by utilizing a priori information on the optimal location of the corresponding functional brain area on the opposite hemisphere. Technique 3) The weighted mean of the study population locations (Eq. (9) by utilizing a priori information on the optimal locations of all the other functional brain areas.

The analysis was performed using full leave-one-out cross-validation: the subject representing the target subject was excluded from the study population, and the probabilistic model was generated using the remaining subjects. This was repeated for each one of the 56 study population subjects. As a result, the probabilistic brain models used in this paper were based only on 55 subjects and were slightly different for each target subject. 


\section{Results}

The results for the estimation accuracy of the locations of the functional brain areas were $8.8 \pm 4.3 \mathrm{~mm}, 9.2 \pm 5.2 \mathrm{~mm}, 8.7 \pm 5.2 \mathrm{~mm}$ and $8.8 \pm 4.9 \mathrm{~mm}$ for opponens pollicis (right hemisphere), opponent pollicis (left hemisphere), tibialis (right hemisphere) and tibialis (left hemisphere), respectively. These numbers correspond to the Technique 1 . The error was reduced by $0.5-1.5 \mathrm{~mm}$ by utilizing the information on the optimal locations of the other functional brain areas located using NBS (Techniques 2 and 3). The best result was obtained by utilizing all the other functional brain areas (Technique 3) although the difference compared with the Technique 2 was small.

Examples of the distributions of the functional brain areas on a subjectspecific head model using the navigation tool are shown in Fig. 2. In this example, the optimal locations of all the functional brain areas already obtained using NBS were utilized to provide a priori information.
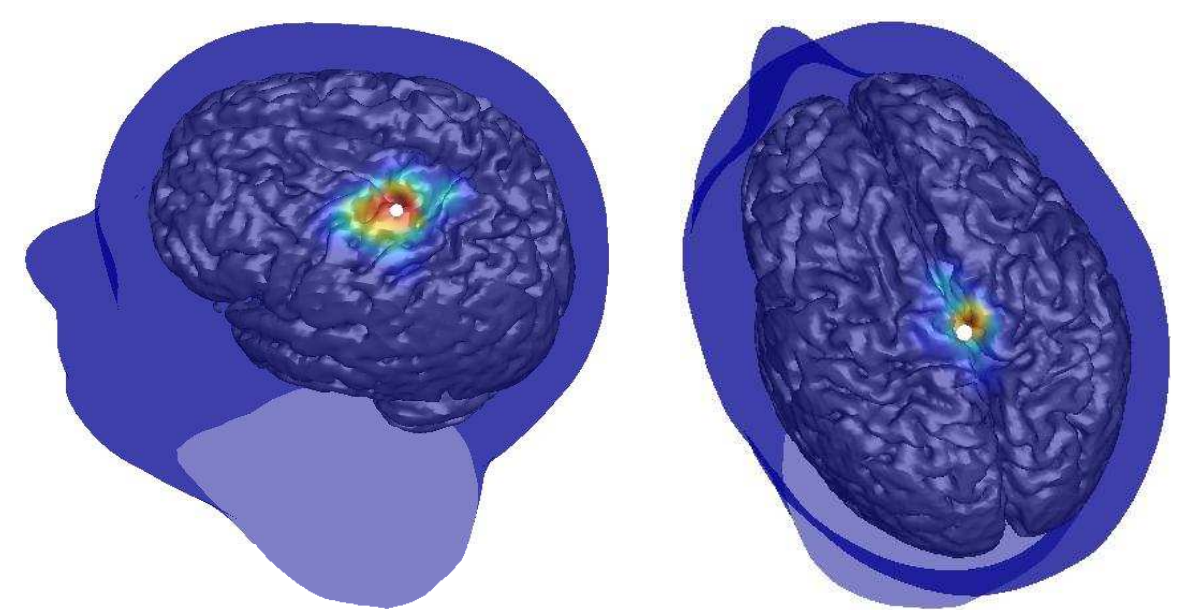

Fig. 2. Probability distributions for the two functional brain areas visualized on a subject-specific head model

\section{Discussion}

In the study described in this paper, a fast, accurate, and low-cost method was developed for NBS studies to provide an estimate of the locations of functional brain regions using a dynamic probabilistic atlas. In addition, methods for the visualization of the distributions of the functional brain areas on a subject-specific head model were developed. These methods enable reasonably accurate identification of the desired NBS target sites even without individual brain imaging results.

The accuracy of the estimates of the locations of the functional brain areas obtained using the probabilistic brain model was about nine millimeters. The 
estimation accuracy was better for the representation area of the tibialis than for the representation area of the opponens pollicis. Especially, the utilization of the localization information from the opposite hemisphere improved much more the localization accuracy of the representation area of the tibialis. This was because the representation area of the tibialis is located close to the mid-sagittal plane, and therefore, the distance to the opposite hemisphere is short. We have tested the utilization of the MR images in the registrations needed in the construction of the probabilistic brain model, but this has not improved the accuracy of the estimates.

The developed methods were fast. The non-rigid point-based registration of the mean brain template to the target subject head took approximately $3-4$ seconds (implemented with $\mathrm{C}$ ), the computation of the estimate of the location of a functional brain area less than one second (implemented with Matlab), and the computation of the distribution of the location of a functional brain area approximately eight seconds (implemented with Matlab) using a standard PC workstation. Especially the parts implemented with Matlab could be further hastened.

\section{References}

1. Barker, A., Jalinous, R., Freeston, I.: Non-invasive magnetic stimulation of human motor cortex. Lancet. 1(8437), 1106-1107 (1985)

2. Hallett, M.: Transcranial magnetic stimulation and the human brain. Nature 406, 147-150 (2000)

3. George, M., Nahas, Z., Molloy, M., Speer, A., Oliver, N., Li, X.B., Arana, G., Risch, S., Ballenger, J.: A controlled trial of daily left prefrontal cortex TMS for treating depression. Biological Psychiatry 48(10), 962-970 (2000)

4. Pridmore, S., Kleinjung, T., Langguth, B., Eichhammer, P.: Transcranial magnetic stimulation: Potential treatment for tinnitus? Psychiatry and Clinical Neurosciences 60(2), 133-138 (2006)

5. Hannula, H., Ylioja, S., Pertovaara, A., Korvenoja, A., Ruohonen, J., Ilmoniemi, R., Carlson, S.: Somatotopic Blocking of Sensation with Navigated Transcranial Magnetic Stimulation of the Primary Somatosensory Cortex. Human Brain Mapping 26(2), 100-109 (2005)

6. Koikkalainen, J., Lötjönen, J.: Reconstruction of 3-D Head Geometry from Digitized Point Sets: an Evaluation Study. IEEE Transactions on Information Technology in Biomedicine 8(3) (2004)

7. Arun, K., Huang, T., Blostein, S.: Least-squares fitting of two 3-D point sets. IEEE Transactions on Pattern Analysis and Machine Intelligence 9(5), 698-700 (1987)

8. Rueckert, D., Sonoda, L., Hayes, C., Hill, D., Leach, M., Hawkes, D.: Nonrigid Registration Using Free-Form Deformations: Application to Breast MR Images. IEEE Transactions on Medical Imaging 18(8), 712-721 (1999)

9. Duda, R., Hart, P.: Pattern Classification and Scene Analysis. John Wiley \& Sons, New York (1973) 